\title{
HEMOGLOBINA MATERNA EN LA SALUD PERINATAL Y MATERNA EN LA ALTURA: IMPLICANCIAS EN LA REGIÓN ANDINA
}

\author{
Gustavo F. Gonzales $1,2, a$
}

\section{RESUMEN}

Esta revisión analiza la importancia del valor de la hemoglobina en la gestante, y su implicancia sobre el embarazo. Discute el uso de combustible de biomasa y la repercusión en el peso del recién nacido, los valores de hemoglobina materna y el riesgo de muerte fetal tardía, parto pretérmino y nacer pequeño para la edad gestacional. Asimismo, se aborda la necesidad de corregir el punto de corte de los valores de hemoglobina para definir anemia en la altura. La evidencia actual sugiere no corregirla, así también, la suplementación de hierro a las gestantes debería ser dirigida a los casos de anemia moderada o severa. Se discute si se debería reorientar la suplementación de hierro a niños de 6 a menos de 36 meses de edad

Palabras clave: Recién nacido de bajo peso; Recién nacido pequeño para la edad gestacional; Anemia; Eritrocitosis; Muerte fetal (fuente: DeCS BIREME).

\section{MOTHER'S HEMOGLOBIN IN PERINATAL AND MOTHER HEALTH IN THE HIGHLANDS: IMPLICATIONS IN THE ANDEAN REGION}

\section{ABSTRACT}

This review analyzes the importance of hemoglobin levels in pregnant women and its implications in pregnancy. It discuss the use of biomass fuel for cooking and the impact on birthweight, maternal hemoglobin levels and the risk of late fetal death, pre-term delivery, and small for gestational age. Furthermore, the need to correct the cutoff points of hemoglobin level to define anemia at high altitudes is addressed. Current evidence suggests that corrections should not be made and iron supplements should be given to pregnant mothers with moderate or severe anemia. It is discussed whether iron supplementation should change its target population from pregnant women to infants aged 6 to 36 months.

Key words: Infant, low birth weight; Infant, small for gestational age; Anemia; Erythrocytosis; Fetal death (source: MeSH NLM).

\section{INTRODUCCIÓN}

El Perú presenta características particulares en cada una de sus tres regiones geográficas. La vida en la sierra implica una serie de condiciones que la hacen diferente a lo que acontece en costa o selva. Entre ellos, el ambiente adverso y la pobreza condicionan el elevado uso de combustible de biomasa (leña, champa, bosta) para cocinar, lo cual produce gran contaminación dentro de las casas, agudiza el problema de la hipoxia e incrementa los niveles de hemoglobina $(\mathrm{Hb})$ observados en la altura.

Ello determina que la tasa de muerte fetal tardía (MFT), parto pretérmino, nacidos pequeños para edad gestacional (PEG), preeclampsia, y mortalidad neonatal y materna sean más altas en la altura (1). Esto ocurre a pesar de que los pobladores de la selva presentan peores índices de pobreza que en la sierra ${ }^{(2)}$, lo que indicaría que existen factores aún no estudiados en la altura que podrían influir en el mayor número de casos. La presente revisión analiza la información existente sobre hemoglobina materna en la altura y su impacto en la salud de la madre y del recién nacido.

\section{CARACTERÍSTICAS DEL RECIÉN NACIDO EN LA ALTURA}

El peso del recién nacido en la altura de Perú es menor que a nivel del mar y disminuye conforme aumenta la altitud (Figura 1); sin embargo, en las poblaciones de mayor antigüedad de residencia en la altura, como las

\footnotetext{
Unidad de Reproducción Humana, Instituto de Investigaciones de la Altura, Universidad Peruana Cayetano Heredia. Lima, Perú.

2 Departamento de Ciencias Biológicas y Fisiológicas, Facultad de Ciencias y Filosofía, Universidad Peruana Cayetano Heredia. Lima, Perú.

a Médico endocrinólogo, doctor en Medicina, doctor en Ciencias

Recibido: 20-07-12 Aprobado: 14-11-12
}

Citar como: Gonzales GF. Hemoglobina materna en la salud perinatal y materna en la altura: implicancias en la región andina. Rev Peru Med Exp Salud Publica. 2012;29(4):570-74. 


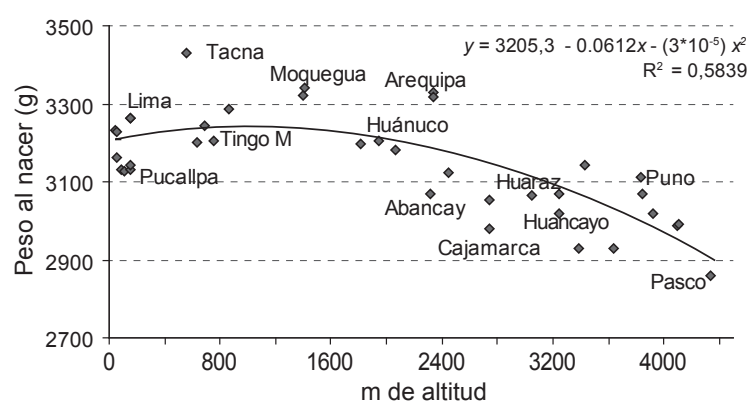

Figura 1. Peso al nacer en ciudades ubicadas a diferentes niveles de altitud en Perú. Fuente: Gonzales, no publicado.

aymaras de Puno, el peso del recién nacido es mayor que en los andes centrales ${ }^{(3)}$. Asimismo, presentan una tasa menor de MFT y valores de hemoglobina materna más bajos debidos a la adaptación a la altura y no por ser anémicos ${ }^{(4,5)}$. La MFT en las grandes alturas (> $3000 \mathrm{~m}$ ) de Perú es 4,8 veces más frecuente que a nivel del mar ${ }^{(4)}$.

Una situación similar se observa entre los tibetanos y los de la etnia han, ambos grupos viven en los Himalayas, pero los primeros viven en ese lugar hace 25000 años y los últimos solo 60 años ${ }^{(6)}$. Los tibetanos recién nacidos muestran mayor peso asociado a menores niveles de Hb materna ${ }^{(7)}$.

\section{COMBUSTIBLE DE BIOMASA Y PESO AL NACER}

Un tercio de la población peruana reside en altura y en un tercio de la viviendas (dos millones de viviendas) se utiliza combustible de biomasa para cocinar, hecho que produce efectos desfavorables en el peso del recién nacido cuando la madre es expuesta durante la gestación ${ }^{(8,9)}$. Ello lleva a que, a la reducción del peso al nacer observado por efecto de la altura ${ }^{(3)}$, se produzca una reducción adicional por las condiciones al cocinar.

\section{HEMOGLOBINA MATERNA Y EL RECIEN NACIDO}

En altura la población se caracteriza por tener niveles aumentados de $\mathrm{Hb}$ por efecto de la hipoxia (10). Recientemente se ha analizado una base de datos de 446397 gestantes y sus partos atendidos desde el 2000 al 2010 en 43 unidades de maternidad pública en 37 provincias del Perú. Se evaluó si los diferentes niveles de $\mathrm{Hb}$ y grados de anemia materna (leve, moderada y severa) tenían asociación con los resultados perinatales o maternos adversos en poblaciones a baja (1000 $1999 \mathrm{~m})$, moderada (2000 $-3000 \mathrm{~m})$ y gran altitud del Perú $(>3000 \mathrm{~m})^{(5,11-14)}$.
Los resultados mostraron que la anemia materna leve $(\mathrm{Hb} \geq 9$ y $<11 \mathrm{~g} / \mathrm{dL})$ no afecta al feto ni a la madre a cualquier nivel de altitud o trimestre de gestación. En tanto que las gestantes con $\mathrm{Hb}<9,0 \mathrm{~g} / \mathrm{dL}$ (anemia moderada y severa) tienen mayor riesgo de MFT, parto pretérmino, y de nacer PEG, con efectos similares a nivel del mar, altitud moderada ${ }^{(12)}$ y en las grandes alturas (11). Igualmente, demuestran que niveles de $\mathrm{Hb}>13,5 \mathrm{~g} / \mathrm{dL}$ se asocian con efectos desfavorables en el recién nacido, particularmente en el peso; y que vivir a moderada o gran altitud tiene un efecto negativo sobre el recién nacido independiente del nivel de $\mathrm{Hb}$, lo cual significa que asociar el efecto de la altura a valores altos de $\mathrm{Hb}$ materna era doblemente negativo en la salud del feto ${ }^{(11)}$. La Hb óptima para el menor riesgo de nacer PEG en el Perú se encuentra en el nivel definido como anemia leve, que corrobora lo demostrado en poblaciones a nivel del mar ${ }^{(15,16)}$.

Con estos resultados se cuestiona la necesidad de dar suplemento de hierro a todas las gestantes al tener en cuenta que valores de $\mathrm{Hb}>13,5 \mathrm{~g} / \mathrm{dL}$ se asocian con tasas altas de recién nacidos PEG ${ }^{(11,12)}$, y que otros autores demuestran que mujeres no anémicas que recibieron suplemento de hierro y aumentaron la $\mathrm{Hb}$ por encima de 14,5 g/dL presentaron mayores tasas de hijos PEG ${ }^{(17,18)}$. Más aun, el administrar suplemento de hierro de manera diaria a gestantes no anémicas produce estrés oxidativo ${ }^{(19)}$ y mayor peroxidación lipídica ${ }^{(20)}$.

Igualmente, el riesgo de preeclampsia aumenta con valores de $\mathrm{Hb}$ materna $>14,5 \mathrm{~g} / \mathrm{dL}$ o $<7,0 \mathrm{~g} / \mathrm{dL}$ y que el riesgo de hemorragia posparto aumenta con la anemia moderada o severa, y que la anemia leve a cualquier nivel de altitud no estuvo asociada con riesgo de preeclampsia ni de hemorragia posparto. La mortalidad materna se incrementa con la anemia moderada o severa y por la eritrocitosis $(\mathrm{Hb}>14,5 \mathrm{~g} / \mathrm{dL})^{(13)}$.

Gonzales et al. (14) han mostrado que en mujeres gestantes no anémicas en la primera medición de $\mathrm{Hb}$, y que presentan eritrocitosis en la segunda medición ( $\mathrm{Hb}>14,5 \mathrm{~g} / \mathrm{dL})$ la proporción de PEG aumenta al doble, $(19,9 \%)$ que lo observado en las gestantes que se mantienen no anémicas en la segunda medición $(10,5 \%)$ (Figura 2). Si tenemos en cuenta que la tasa de PEG en dos ciudades de moderada altura como Bogotá (Colombia) y Ciudad de México (México) es de 3,6 y $6,0 \%$ respectivamente ${ }^{(21)}$, el valor de $19,9 \%$ por eritrocitosis genera un problema de salud pública. Igualmente, una mujer gestante con $\mathrm{Hb}$ normal en la primera medición pero con un índice de masa corporal (IMC) pregestacional por debajo de lo normal tiene un riesgo alto de hacer anemia moderada o severa en la segunda medición ${ }^{(14)}$. 


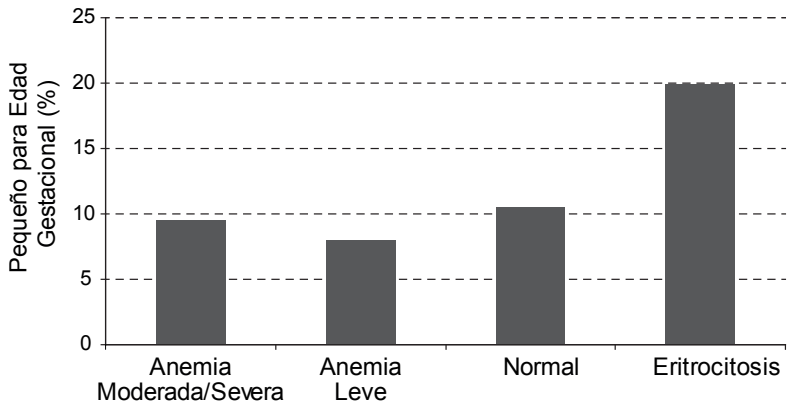

Figura 2. Proporción de pequeño para edad gestacional en gestantes según nivel de $\mathrm{Hb}$ de la gestante.

Nota: primera medición de $\mathrm{Hb}$ dentro del rango de normalidad; la segunda medición se incluyó en la definición de anemia moderada/severa, leve, hemoglobina normal o eritrocitosis ${ }^{(14)}$.

\section{CORRECCIÓN DEL PUNTO DE CORTE PARA DEFINIR ANEMIA EN LAALTURA}

Es conocido que las poblaciones que residen en las alturas muestran niveles más altos de hemoglobina ${ }^{(4)}$. La Organización Mundial de la Salud propuso corregir los puntos de corte de la hemoglobina para definir la anemia por cada nivel de altitud ${ }^{(22)}$. En poblaciones que viven por debajo de $1000 \mathrm{~m}$ de altura, el punto de corte de la $\mathrm{Hb}$ para definir anemia es de $11,0 \mathrm{~g} / \mathrm{dL}^{(23)}$.

Para hacer estas correcciones se han utilizado modelos de regresión y generado ecuaciones matemáticas que permiten tener un valor diferente por cada nivel de altitud ${ }^{(24,25)}$. No hay criterios clínicos en estas definiciones que permitan concluir que cada nivel de $\mathrm{Hb}$ que define la anemia en la altura se asocie con un problema clínico tanto de la madre como del feto. Apunta en contra de corregir el punto de corte el hecho de encontrar en un mismo ambiente altitudinal, a poblaciones con hemoglobina más alta y otra con hemoglobina más baja producto de un proceso de adaptación multigeneracional a la altura y no por situaciones de anemia ${ }^{(5-7)}$.

Una manera de valorar la utilidad de la corrección de la hemoglobina por altitud es comparar la anemia definida por $\mathrm{Hb}$ corregida por altitud con la anemia definida por deficiencia de hierro, midiendo el contenido de hierro corporal. En Bolivia, luego de corregir la $\mathrm{Hb}$ por altitud, existe una prevalencia de anemia de $26,6 \%$, que no concuerda con el resultado al medir contenido de hierro, donde solo el $5,7 \%$ de la misma población tendría anemia ${ }^{(26)}$. Esto implicaría que utilizando correcciones de $\mathrm{Hb}$ para determinar el punto de corte para definir anemia en la altura se estaría sobrevalorando la real prevalencia de la anemia por deficiencia de hierro.

Al corregir el punto de corte de la $\mathrm{Hb}$ por la altura, se incrementa cinco veces la tasa de anemia; sin embargo, cuando se analizan los efectos adversos en el recién nacido (MFT y parto pretérmino) se encuentra más bien que con la corrección del punto de corte de la $\mathrm{Hb}$, las tasas de MFT se reducen de 28,0 por mil nacidos vivos a 18,2 por mil nacidos vivos $(p<0,01)$ y que la tasa de pretérminos (nacidos antes de las 37 semanas) se reduce de 9,4 a $8,2 \%(p<0,01)$ (Gonzales et al., datos no publicados). Estos datos sugieren que no sería necesario corregir el punto de corte de la hemoglobina para definir anemia en la altura.

\section{HIERRO Y HEMOGLOBINA}

El hierro es importante para la salud humana. Sin embargo, su exceso puede ser dañino para el organismo ${ }^{(27)}$. El organismo para evitar que un exceso de hierro pueda ingresar al cuerpo, o que aumenten los niveles circulantes por su salida de los lugares de almacén, regula el ingreso de hierro en los enterocitos del intestino delgado o la salida de los sitios de almacén (hígado, sistema retículo endotelial) a través de la hepcidina, hormona producida por el hígado.

La hepcidina, un péptido de 25 aminoácidos, se une al transportador de hierro, la ferroportina y bloquea su función ${ }^{(28)}$, por lo tanto, la disponibilidad de hierro en la circulación sistémica. Por ello, los mayores requerimientos de hierro se van a regular a través de cambios en los niveles de hepcidina ${ }^{(29)}$. Si se requiere hierro disminuirán los niveles de hepcidina y si hay exceso de hierro aumentarán los niveles de hepcidina.

El hierro se elimina poco y no hay un mecanismo de excreción, de tal manera que las pérdidas diarias oscilan en $2 \mathrm{mg}$, lo cual debe ser repuesto por la ingesta y regulado por la hepcidina quien afectará la capacidad de absorción intestinal. El resto de hierro requerido es reciclado a través del hierro contenido en los macrófagos o en la ferritina contenida en las fuentes de reserva en los tejidos ${ }^{(30)}$. La hemoglobina contiene el $65 \%$ del hierro ${ }^{(31)}$.

En la gestación ocurren cambios que permiten una adecuada disponibilidad de hierro. En el primer trimestre, debido a la organogénesis, la disponibilidad de hierro disminuye pues puede ser tóxico para este proceso ${ }^{(32)}$ y los niveles de hepcidina son altos ${ }^{(33)}$. Luego, conforme las necesidades de hierro aumentan por las necesidades de mayor eritropoyesis y las del feto ${ }^{(34)}$, los niveles de hepcidina disminuyen y aumenta la capacidad de absorción de hierro a nivel intestinal y la movilización del hierro almacenado ${ }^{(35)}$.

Un neonato a término tiene un gramo de hierro que procede de la madre ${ }^{(35)}$. Así, la eritropoyesis materna 
aumenta a partir del segundo trimestre ${ }^{(34)}$, ello da posibilidad a mayor reserva para las necesidades fetales. Esto sin embargo crearía un problema, pues al aumentar la eritropoyesis aumentaría la hemoglobina y produciría un incremento de la viscosidad sanguínea con menor flujo útero-placentario, lo que afectaría el crecimiento del feto ${ }^{(36)}$. Para contrarrestar ello se genera una expansión del volumen plasmático de más de $1,5 \mathrm{~L}{ }^{(36)}$ que es superior al aumento de la masa globular sanguínea y con ello una disminución de la hemoglobina, y una mejora del flujo útero-placentario que favorece el ingreso de nutrientes al feto $y$, por ende, el crecimiento normal del feto ${ }^{(37)}$.

Todo ello determina que los niveles de hemoglobina disminuyan en la gestación como un efecto de esta hemodilución y con la finalidad de mejorar el flujo úteroplacentario. La hemoglobina materna por encima de 13,5 g/dL se asocia a una mayor tasa de nacidos PEG ${ }^{(11,12,14)}$.

\section{REPERCUSIÓN DEL BAJO PESO AL NACER}

Estos estudios tienen una alta repercusión en Medicina Preventiva y Salud Pública pues implicaría la necesidad de replantear el suplemento de hierro solo en situaciones donde definitivamente se requiere, como son los casos de anemia moderada y severa que representan menos del $2 \%$ de la población de gestantes que acuden a hospitales públicos en el Perú ${ }^{(5)}$. Sin embargo, hasta no tener resultados confirmados sobre esta hipótesis, la recomendación que se plantea es utilizar el suplemento de hierro semanal.

El impacto negativo de nacer con bajo peso no solo es inmediato al aumentar las tasas de morbi-mortalidad perinatal o neonatal sino también a largo plazo, pues implican un alto riesgo para tener enfermedades en la vida adulta ampliamente descrita en diferentes partes del mundo ${ }^{(38)}$

\section{COMENTARIOS FINALES}

Este aporte es de importancia también para muchos otros países de América Latina. Así, la población de Bogotá (Colombia), Quito (Ecuador), Puebla, México DF, Estado de México (México), y de Cochabamba, Chuquisaca, La Paz, Oruro, y Potosí (Bolivia) es de 44 807237 habitantes que residen en la altura, mientras que a nivel mundial son alrededor de 140 millones ${ }^{(1)}$. Más aun los problemas de hemoglobina baja o alta también se presentan a nivel del mar por lo que el aporte de estos estudios implica a una amplia población del mundo, por ello el carácter preventivo basado en la vigilancia de la gestación para evitar que una gestante llegue a la anemia moderada o severa $(\mathrm{Hb}<9 \mathrm{~g} / \mathrm{dL})$ o a la eritrocitosis $(\mathrm{Hb}>14,5 \mathrm{~g} / \mathrm{dL})$.

Por otro lado, en el 2010 la proporción de gestantes y menores de 36 meses de edad que recibieron suplemento de hierro en el Perú fue de 86,1 y $18,4 \%$ respectivamente. La tasa de anemia en menores de 36 meses fue de $50,5 \%$ comparado a la baja tasa de anemia $(2,0 \%)$ moderada o severa en las gestantes.

En conclusión, la evidencia científica a la fecha indicaría que: 1) no debe corregirse el punto de corte de los valores de hemoglobina en sangre para definir anemia en la altura; 2) que el suplemento de hierro debería estar dirigido solo a gestantes que hayan sido diagnosticadas como anemia moderada o severa luego de al menos dos mediciones de la hemoglobina; 3 ) los recursos económicos usados para suplementar con hierro a toda gestante sea o no anémica debiera ser reorientados para favorecer el tratamiento de menores de 36 meses con anemia.

Fuentes de financiamiento: autofinanciado.

Conflictos de interés: el autor declara no tener conflictos de interés en la publicación de este artículo.

\section{REFERENCIAS BIBLIOGRÁFICAS}

1. Gonzales GF. Peruvian contributions to the study on human reproduction at high altitude: from the chronicles of the Spanish conquest to the present. Respir Physiol Neurobiol. 2007;158(23):172-9.

2. Instituto Nacional de Estadística e Informática. Perú: Encuesta Demográfica y de Salud Familiar (ENDES) 2011. Lima: INEI; 2012. p. 438.
3. Hartinger S, Tapia V, Carrillo C, Bejarano L, Gonzales GF. Birth weight at high altitudes in Peru. Int J Gynaecol Obstet. 2006;93(3):275-81.

4. Gonzales GF, Tapia V, Carrillo CE. Stillbirth rates in Peruvian populations at high altitude. Int J Gynaecol Obstet. 2008;100(3):221-7

5. Gonzales GF, Tapia V, Gasco M, Carrillo CE. Hemoglobina materna en el Perú: diferencias regionales y efectos perinatales. Rev Per Med Exp Salud Publica. 2011;28(3):484-91.

6. Wu T, Wang $\mathrm{X}$, Wei C, Cheng $\mathrm{H}$, Wang X, Li Y, et al. Hemoglobin levels in Qinghai-Tibet: different effects of gender for Tibetans vs Han. J Appl Physiol 2005;98(2):598-604.

7. Moore LG, Young D, McCullough RE, Droma T, Zamudio S. Tibetan protection from intrauterine growth restriction (IUGR) and reproductive 
loss at high altitude. Am J Hum Biol. 2001;13(5):635-44.

8. Boy E, Bruce N, Delgado H. Birth weight and exposure to kitchen wood smoke during pregnancy in rural Guatemala. Environ Health Perspect. 2002;110(1):109-14

9. Yucra S, Tapia V, Steenland K, Naeher LP, Gonzales GF. Association between biofuel exposure and adverse birth outcomes at high altitudes in Peru: a matched case-control study. Int J Occup Environ Health. 2011;17(4):307-13.

10. Gonzales GF. Hematocrit values in women at high altitude and its relationship with sex hormone levels. Journal of Qinghai Medical College. 2004;25:267-72.

11. Gonzales GF, Steenland K, Tapia V. Maternal hemoglobin level and fetal outcome at low and high altitudes. Am J Physiol Regul Integr Comp Physiol. 2009;297(5):R1477-85

12. Gonzales GF, Tapia V, Gasco M, Carrillo CE. Maternal hemoglobin concentration and adverse pregnancy outcomes at low and moderate altitudes in Peru. J Matern Fetal Neon Med. 2012;25(7):1105-10.

13. Gonzales GF, Tapia V, Gasco M, Carrillo CE, Fort AL. Association of hemoglobin values at booking with adverse maternal outcomes among Peruvian populations living at different altitudes. Int J Gynaecol Obstet 2012;117(2):134-9.

14. Gonzales GF, Tapia V, Fort AL. Maternal and perinatal outcome in second hemoglobin measurement in non anemic women at first booking: effect of altitude of residence in Peru. ISRN Obstet Gynecol. 2012;2012:368571.

15. Steer PJ. Maternal hemoglobin concentration and birth weight. Am J Clin Nutr. 2000;71(5 Suppl):1285S7S. Review

16. Little MP, Brocard P, Elliott P, Steer PJ. Hemoglobin concentration in pregnancy and perinatal mortality: a London-based cohort study. Am J Obstet Gynecol. 2005;193(1):220-6

17. Casanueva E, Viteri FE, MaresGalindo M, Meza-Camacho C, Loría A, Schnaas L, et al. Weekly iron as a safe alternative to daily supplementation for nonanemic pregnant women. Arch Med Res. 2006;37(5):674-82.

18. Peña-Rosas JP, Viteri FE. Effects and safety of preventive oral iron or iron+folic acid supplementation for women during pregnancy. Cochrane Database Syst Rev. 2009; (4):CD004736.

19. Viteri FE, Casanueva E, Tolentino MC, Díaz-Francés J, Erazo AB. Antenatal iron supplements consumed daily produce oxidative stress in contrast to weekly supplementation in Mexican non-anemic women. Reprod Toxicol. 2012;34(1):125-32.

20. Bhatla N, Kaul N, Lal N, Kriplani A, Agarwal N, Saxena R, et al. Comparison of effect of daily versus weekly iron supplementation during pregnancy on lipid peroxidation. J Obstet Gynaecol Res. 2009;35(3):438-45.

21. Boguszewski MCS, Mericq V, Bergada I, Damiani D, Belgorosky A, Gunczler $\mathrm{P}$, et al. Latin American consensus: children born small for gestational age. BMC Pediatrics. 2011;11:66.

22. World Health Organization. Prevalence of anaemia in women. In: Reproductive Health Indicators. Guidelines for their generation, interpretation and analysis for global monitoring. Geneva: WHO; 2006. p. 41-3.

23. WHO/NHD. Iron Deficiency Anaemia: Assessment, Prevention and Control. A Guide for Programme Managers. Geneva: WHO; 2001.

24. Dallman PR, Siimes MA, Steckel A. Iron deficiency in infancy and childhood. Am J Clin Nutr. 1980;33:86-118.

25. Cohen JH, Haas JD. Hemoglobin correction factors for estimating the prevalence of iron deficiency anemia in pregnant women residing at high altitudes in Bolivia. Rev Panam Salud Publica. 1999;6(6):392-9.

26. Cook JD, Boy E, Flowers C, Daroca Mdel C. The influence of highaltitude living on body iron. Blood. 2005;106(4):1441-6.

27. Zaccone V, Gasbarrini G. From iron accumulation to organ damage. Minerva Med. 2012;103(2):123-40.

28. Ganz T, Nemeth E. The hepcidinferroportin system as a therapeutic target in anemias and iron overload disorders. Hematology Am Soc Hematol Educ Program. 2011;2011:538-42.

29. Ganz T, Nemeth E. Hepcidin and iron homeostasis. Biochim Biophys Acta. 2012;1823(9):1434-43.

30. Kogho Y, Ikuta K, Ohtake T, Torimoto Y, Kato J. Body iron metabolism and pathophysiology of iron overload. Int J Hematol. 2008;88(1):7-15.

31. Muñoz M, Villar I, García-Arce JE. An update on iron physiology. World J Gastroenterol. 2009;15(37):4617-26.

32. Weinberg ED. First trimester curtailment of iron absorption: innate suppression of a teratogen? Med Hypotheses. 2010;74(2):246-7.

33. Evans P, Cindrova-Davies T, Muttukrishna S, Burton GJ, Porter J, Jauniaux E. Hepcidin and iron species distribution inside the first-trimester human gestational sac. Mol Hum reprod. 2011;1(4)7:227-32.

34. Choi JW, Pai SH. Change in erythropoiesis with gestational age during pregnancy. Ann Hematol. 2001;80(1):26-31.

35. Cetin I, Berti C, Mandò C, Parisi F. Placental iron transport and maternal absorption. Ann Nutr Metab. 2011;59(1):55-8.

36. von Tempelhoff GF, Velten E, Yilmaz A, Hommel G, Heilmann L, Koscielny J. Blood rheology at term in normal pregnancy and in patients with adverse outcome events. Clin Hemorheol Microcirc. 2009;42(2):127-39.

37. Ferrazzi E, Rigano S, Padoan A, Boito S, Pennati G, Galan HL. Uterine artery blood flow volume in pregnant women with an abnormal pulsatility index of the uterine arteries delivering normal or intrauterine growth restricted newborns. Placenta. 2011;32(7):487-92.

38. Ozanne SE, Fernandez-Twinn D, Hales $\mathrm{CN}$. Fetal growth and adult diseases. Semin Perinatol. 2004;28(1):81-7.

Correspondencia: Gustavo F. Gonzales Dirección: Av. Honorio Delgado 430,

Lima 31, Perú.

Teléfono: (511) 3190000 anexo 2535

Correoelectrónico:gustavo.gonzales@upch.pe 